\title{
Acetyleugenol from Acacia nilotica (L.) exhibits a strong antibacterial activity and its phenyl and indole analogs show a promising anti-TB potential
}

\author{
Abubakar Abdulhamid 1, Talal A. Adlan ${ }^{2}$, Abdalla E. Ahmed ${ }^{3}$, Faisal Hammad Mekky Koua, ${ }^{4, *}$ and Amar \\ Mohamed Ismail ${ }^{5, *}$ \\ 1 Department of Biochemistry, Faculty of Science, Kebbi State University of Science and Technology, PMB 1144, Aliero, \\ Kebbi State, Nigeria.; abuaugie132@yahoo.com \\ 2 Ibn-Sina University, Aljerif West Bank Block 5, PO Box 10995, Khartoum, Sudan.; talaladlan@hotmail.com \\ 3 National University Biomedical Research Institute, National University-Sudan, Air St. PO Box 3783, Khartoum, Sudan.; \\ faisal.koua@cfel.de; fkoua@nuri.nu.edu.sd \\ 4 Biotechnology Park, African City of Technology, Bahri Central Blue Nile Ave, Khartoum 11111, Sudan.; \\ abdallah_elseer@yahoo.com \\ ${ }_{5}$ Department of Biochemistry and Molecular Biology, Faculty of Science and Technology, Al Neelain University, El \\ Baladiya Ave, PO Box 12702, Khartoum, Sudan.; amarqqqu@yahoo.com
}

* Correspondence: faisal.koua@cfel.de; Tel.: +49-40-8998-6204 (F.H.M.K.) or amarqqqu@yahoo.com (A.I.)

Received: date; Accepted: date; Published: date

\begin{abstract}
Acetyleugenol is a phytochemical compound with broad effect against infectious diseases and tumors. Here, we extracted, characterized and elucidated the structure of acetyeugenol, for the first time, from the leaves of Acacia nilotica (L.) - a well-known medicinal plant. The broad antibacterial potential of acetyleugenol was first confirmed against seven bacterial pathogenic isolates with best activity against Proteus sp., Salmonella typhi, Staphylococcu aureus, and Streptococcus pneumonia, which showed similar or better zone of inhibition to that of the control amoxicillin. To further investigate its effect against Mycobacterium tuberculosis, acetyleugenol and its indole and phenyl analogs were subjected to molecular docking experiments against two potential tuberculosis drug targets- $M t P k n E$ and $M t P k n B$ Ser/Thr protein kinases. The results reveal that all of the analogs have improved docking scores comparing to the acetyleugenol. The indole analogs EUG-1 and EUG-3 were more effective with better docking scores for MtPknE with -11.08 and $-10.05 \mathrm{kcal} / \mathrm{mol}$, respectively. Similar results were obtained for the MtPknB. In contrast, only the EUG-2 phenyl analog has given rise to similar docking scores for both targets. This opens the door for further comprehensive studies on these acetyleugenol analogs with in vitro and in vivo experiments to validate and get more insights into their mechanisms of action.
\end{abstract}

Keywords: acetyleugenol; Acacia nilotica; anti-tuberculosis; Serine/Threonine kinases; multi-drug resistant TB.

\section{Introduction}

Tuberculosis (TB) caused by Mycobacterium tuberculosis is among the top 10 causes of death worldwide [1], and represents serious challenges that faces the global disease eradication policy owing to the growing antimicrobial resistance [2,3]. TB kills annually around 1.5 million people with over $80 \%$ in the developing countries including India, China, Indonesia, Philippines, Pakistan, Nigeria, Bangladesh and South Africa [4]. TB treatment regimen is a complex procedure that involves the use of several antibiotics for a long-period of time and requires special awareness from patients. The lack of such awareness leads often to the emergence of multi-drug resistant tuberculosis (MDR-TB), which is a consequence of the lack of good education and poor hygienic condition and other environmental factors especially in developing countries [5]. This has also increases the toll of death due to TB among HIV-positive patients [6,7]. The drug resistance developed by $M$. tuberculosis against first line anti-tuberculosis (anti-TB) drugs-Isoniazid and Rifampicin, has become a major problem worldwide [8-10]. This has been complicated by the emergence of resistance against other anti-TB 
drugs including, for example, the quinolones and aminoglycosides [11,12]. The emergence of the MDR-TB has driven the needs for the discovery of novel therapeutic agents to overcome the TB challenge worldwide [9-12].

Medicinal plants are considered a major source in drug discovery which have well-documented applications as natural remedies for the treatment of many infectious diseases including those infecting the respiratory system [13]. Plants produce many secondary metabolites in tremendous amounts and many of which have been used as templates or scaffolds for synthesis of novel pharmaceutical drugs [13-15]. In the developing countries plants remain the major source of medicines for centuries [16,17]. Sub-Saharan Africa is rich in medicinal plants, which have been used extensively to treat infectious diseases especially those of the respiratory system such as tuberculosis, asthma, influenza and pneumonia [17]. For example, in South Africa alone there are over 300 medicinal plants, with $\sim 6 \%$ species belong to the family Fabaceae, that have been used in remedies of various respiratory infections [18]. The medicinal plant Acacia nilotica (L.) 一native to Sudan-from to the family Fabaceae is well-known for its medicinal applications in the treatment of various respiratory system diseases and other diseases including malaria and Hepatitis $C$ virus as well as wound healing and disinfection [19-21]. A. nilotica (L.) is rich with several groups of active phytochemical constituents including alkaloids, flavonoids, tannins and saponins, gallic acid, kaempferol, umbelliferone, and niloticane [22-24]. Its pharmacological activities are broad-mostly attributed to these phytochemicals-covering antibacterial, antifungal, anti-mutagenic, anti-inflammatory and antioxidant activities [20,22,25].

Eugenol, 4-allyl-2-methoxybenzene, is a natural product that is produced by many angiosperms and known for its aromatic flavor [26,27]. It is a phenolic compound that belongs to the group of phenylpropanes, which is biosynthesized via the shikimic acid pathway [27]. Its derivative acetyleugenol, with the formula $\mathrm{C}_{12} \mathrm{H}_{14} \mathrm{O}_{3}$ and molecular weight $206.238 \mathrm{Da}$, is one of the major components of clove Syzygium aromaticum (L.) essential oil and is also produced by many other plants such as cinnamon, pimento, ylang ylang and rose [28]. Eugenol has also been detected in the chloroform extracts of the A. nilotica (L.) leaves without further chemical description [29]. Eugenol is slightly acidic and very reactive with a stronger action than alcohols [28]. It has a wide range of applications in food and cosmetic industry as flavor and antioxidant as well as in the traditional medicine [26,28,30]. Eugenol and its derivatives have also been reported to have potential activities as antiinflammatory, anti-tumor, antimicrobial, anti-pyretic, analgesic and anesthetic [31]. Its benzoate and 2nitrobenzoate derivatives as well as its synergistic effects with approved anti-TB drugs have been shown to exhibits strong activity against Mycobacterium spp. and M. tuberculosis [32]. Its hydrazone derivatives were studied by molecular docking against the enoyl-ACP reductase as a molecular target and validated by in vitro assay which showed a docking score of $-10.393 \mathrm{kcal} / \mathrm{mol}$ with minimum inhibitory activity at $25 \mu \mathrm{g} / \mathrm{ml}$ [31,33].

The M. tuberculosis Serine/Threonine (S/T) protein kinases MtPnkA-L, except PknC, are considered as potential anti-TB drug targets [34]. The M. tuberculosis S/T protein kinases, belong to the Pkn2 family, are eukaryote-like S/T kinases and function in phosphorylation-dependent signal transduction manner by transducing extracellular stimuli into a cellular response to ensure $M$. tuberculosis cell division, transcription regulation, stress response, regulation of multiple metabolic processes and pathogenesis [34,35]. These $M t$ kinases can be categorized based on their similarity into five clades; Clade-1 (PknA, PknB, PknL), Clade-2 (PknH, PknE, PknD), Clade-3 (PknF, PknI, PknJ), Clade-4 (PknK) and Clade-5 (PknG) [36]. MtPknB and $M t P k n E$ have been implicated in important biological roles in $M$. tuberculosis which include the determination of cell shape, morphology, cell division and biofilm formation for PknB [37,38], and inhibition of apoptosis in infected macrophages for PknE [39,40]. Thus, both PknB and PknE are considered as important molecular targets for effective anti-TB drug search [34,41,42]. The $M t P k n B$ and $M t P k n E$ crystal structures were reported previously and their structures have been used successfully as molecular targets for inhibitors screening and drug discovery [43-45]. Here in, we applied bioactivity-guided screening of acetyleugenol isolated from $A$. nilotica (L.) leaves against various pathogenic bacterial isolates and performed molecular docking studies using $M t P k n B$ and $M t P k n E$ as targets. Based on the preliminary results of isolated acetyleugenol, we designed and in silico synthesized phenyl and indole derivatives using acetyleugenol as a chemical scaffold and studied them with docking experiments against the same targets.

\section{Materials and Methods}




\subsection{Plant collection and authentication}

Fresh disease-free leaves of A. nilotica (L.) were separately collected from Bodinga, Sokoto State, Nigeria. The specimens were identified and authenticated by Botanists at the Department of Biological Sciences, Usmanu Danfodiyo University, Sokoto, Nigeria. The specimens identified as A. nilotica (L.) has given the voucher number UDUH/ANS/0247. Specimens were then shed-dried, ground finely and kept in air-tight containers until further use.

\subsection{Preparation of ethyl acetate extracts}

Crude methanolic extraction was initially performed followed by solvent partitioning on the residue (marc) to obtain the ethyl acetate extract. In brief, the methanol extraction was carried out by soaking $1 \mathrm{~kg}$ of the specimen in $6 \mathrm{~L}$ of $90 \%$ methanol for $72 \mathrm{~h}$ and was filtered using a clean cloth and Whatman No. 1 filter paper. The filtrate was then concentrated in vacuo at $30^{\circ} \mathrm{C}$ and stored in sterile containers at $4^{\circ} \mathrm{C}$ until further use. For the solvent partitioning, about $100 \mathrm{~g}$ of the crude extract of A. nilotica was dissolved in $500 \mathrm{ml}$ sterile distilled water in a separatory funnel and extracted with $n$-hexane. The resulting $n$-hexane phase was concentrated to dryness and the powder was kept in a freezer in an air-tight container. The aqueous phase was further extracted with ethyl acetate. The fraction obtained from the ethyl acetate extraction was concentrated to dryness and the powder was kept at $4^{\circ} \mathrm{C}$ until used. The same procedure was applied to obtain the $n$-butanol fraction.

\subsection{Isolation and purification of the bioactive compounds}

Thin layer chromatography (TLC), column chromatography (CC) and preparative TLC techniques were used for the isolation and purification of the bioactive compounds as described previously [46,47].

\subsection{Thin Layer Chromatography}

TLC was carried out on aluminum TLC sheets pre-coated with silica gel (60 PF254) with a layer thickness of $\sim 0.2 \mathrm{~mm}$. The one-way ascending spotting and development technique was followed. Spots were applied manually on silica gel aluminum plates using a capillary tube and dried using an air blower. The TLC was developed at a room temperature using a Shandon chromatographic tank covered with a lid during the run. The solvent system contained a mixture of $n$-hexane and ethyl acetate solvent with a ratio of 70:30 (see Figure $\mathrm{S} 1)$.

\subsection{Column Chromatography}

The ethyl acetate extract was loaded on a stationary phase containing silica gel (60-120 mesh size) using the wet loading method in a glass column $(75 \times 3.5 \mathrm{~cm}$ dimensions) packed with a wet slurry silica gel [46]. The extract was first dissolved in a small amount of $n$-hexane mixed with a small quantity of silica gel and then loaded on top of the pre-packed column. Different solvent systems were used to elute the silica gel column by gradient elution method-i) 100\% $n$-hexane, ii) $n$-hexane/ethyl acetate mixtures (5:95, 10:90, 15:85, 20:80, 25:75, 30:70, 35:65, 40:60, 45:55, 50:50, 55:45; 60:40, 65:35, 70:30, 75:25, 80:20, 85:15, 90:10, 95:5), iii) 100\% ethyl acetate, or iv) ethyl acetate/methanol mixtures with similar ratios to that of $n$-hexane/ethyl acetate. The best solvent system from which the fraction was eluted is $n$-hexane/ethyl acetate (75:25), which was confirmed by TLC using $n$-hexane/ethyl acetate solvent with 70:30 ratio.

The ethyl acetate extract-the most effective in the anti-bacterial sensitivity assays—was further subjected to CC fractionation as described above. In brief, $3.0 \mathrm{~g}$ of the extract was chromatographed on a silica gel column eluted with a mobile phase containing; i) $100 \% n$-hexane, ii) $n$-hexane/ethyl acetate mixtures (90:10, 80:20; 70:30, 60:40, 50:50, 40:60, 30:70, 20:80, 10:90, iii) 100\% ethyl acetate, or iv) ethyl acetate/methanol mixtures (98:20, 95:5, (0:10, 80:20), which were gave rise to a total of 110 fractions. These 110 fractions were pooled together based on similarity in their TLC profile and their retention factors $\left(\mathrm{R}_{\mathrm{f}}\right)$, calculated according to equation (1), to give rise into 5 sub-fractions. The sub-fractions were then subjected to TLC and a single spot was observed for each fraction confirming the similarity and assuring the pooling of sub-fractions. Subfractions were dried, weighed and labelled before used for bioactivity assay. 


$$
R_{f}=\frac{\text { Distance traveled by spot }}{\text { Distance traveled by solvent }}
$$

equation (1)

\subsection{Structural elucidation of the bioactive compounds}

The structures of the bioactive compounds based on the antibacterial assays were elucidated and characterized by different spectroscopic and spectrometry methods including Gas chromatography/mass spectrometry (GC/MS), nuclear magnetic resonance (NMR), Fourier Transform-Infrared spectroscopy (FT-IR) and UV-visible spectrophotometry.

\subsection{Gas Chromatography/Mass Spectrometry analysis}

For GC/MS analysis, $0.5 \mathrm{mg}$ of each pure compound was placed in a labelled vial and diluted with an appropriate solvent of HPLC grade up to a final concentration of $1.0 \mu \mathrm{M}$. The solution in each case was filtered to remove any insoluble material before injecting into the column. An aliquot of $2.0 \mathrm{ml}$ of each sample solution was then injected into a GC/mass spectrometer equipped with a GC-MSD detector (Agilent GC 7890B, MSD 5977A, Agilent Technologies, USA) and ran for $27 \mathrm{~min}$. The column oven and injection temperature were set at $80^{\circ} \mathrm{C}$ and $250^{\circ} \mathrm{C}$, respectively, and the column pressure was maintained at $108 \mathrm{kPa}$ during the run. The spectra were analyzed using the program GCMS-QP2010 PLUS.

\subsection{Nuclear Magnetic Resonance spectroscopy}

Proton $\left({ }^{1} \mathrm{H}\right)$ and carbon-13 isotope $\left({ }^{13} \mathrm{C}\right)$ NMR experiments alongside two-dimensional (2D) NMR spectroscopy including correlation spectroscopy (COSY), nuclear Overhauser effect Spectroscopy (NOESY), heteronuclear single-quantum correlation spectroscopy (HSQC), and heteronuclear multiple-bond correlation spectroscopy (HMBC) were employed to get structural information on the isolated compounds. For each sample, $10 \mathrm{mg}$ of the pure compound was dissolved in $1.0 \mathrm{ml}$ of $100 \%$ dimethyl sulfoxide (DMSO, SigmaAldrich) as a solvent and filtered through a Pasteur pipette equipped with a glass wool plug that was discharged into a labelled $5.0 \mathrm{~mm}$ NMR tube. The filtration serves to remove undissolved residues and dust from the solution that affects the resolution and line shape of NMR spectra. NMR spectra were recorded on 400 MHz Agilent-VNMRS-NMR spectrometer (Agilent Technologies, USA). Chemical shift values were reported in parts per million (ठ-ppm). All spectra were analyzed and the results obtained were compared with published data in order to elucidate the structures of the isolated compounds.

\subsection{Bioactivity antibacterial sensitivity assays}

\subsubsection{Microorganisms, culture media and inoculum preparations}

The microorganisms used in the present study were locally isolated and identified by the Department of Microbiology, Faculty of Science, Kebbi State University of Science and technology, Aliero, Nigeria. All isolates were purified and maintained in slant cultures of Mueller Hinton Agar (MHA). These isolates are Escherichia coli, Klebsiella pneumoniae, Proteus sp., Pseudomonas aeruginosa, Staphylococcus aureus, Salmonella typhi and Streptococcus pneumonia. The sensitivity tests were performed on either Mueller Hinton Agar or Mueller Hinton Broth (MHB), which were all prepared freshly under aseptic condition.

To prepare the inoculum of each microorganism a 0.5 McFarland turbidity standard was used to standardize the microorganism. The scale was prepared by adding $50 \mu \mathrm{l}$ of $1 \% \mathrm{BaCl}_{2}$ to $9.95 \mathrm{ml}$ of $1 \% \mathrm{H}_{2} \mathrm{SO}_{4}$. The suspensions of the microorganisms were prepared in normal saline and compared with 0.5 McFarland turbidity standard against a white background with contrasting black lines in the presence of good lighting [48]. The suspensions were diluted with normal saline if the density is higher than that of the standard and additional microorganism suspension was added in case of lower density. This continues until the density of the suspension matches with that of $0.5 \mathrm{McFarland}$ turbidity standard, which corresponds to $\sim 1.5 \times 10^{18}$ $\mathrm{CFU} / \mathrm{ml}$.

\subsubsection{Antibacterial sensitivity assay of the extracts}


Agar well diffusion method was used to test the antibacterial activity of column fractions as reported previously [49,50]. The assay was performed using stock concentrations of $10 \mathrm{mg} / \mathrm{ml}$. The standardized inoculums of the isolates were uniformly inoculated on a freshly prepared MHA plates by streaking the swab extensively over the entire surface of the plates. A sterile Cork borer of $6.0 \mathrm{~mm}$ diameter was used to make two wells per plates and labelled appropriately. An aliquot of $20 \mu \mathrm{l}$ of the extract was added in the well and allowed to diffuse into the agar and incubated at $37^{\circ} \mathrm{C}$ for $24 \mathrm{~h}$. The zone of inhibition diameter was measured in millimeter $(\mathrm{mm})$ as an indication of antibacterial activity of the extract against the bacterial isolate. The antibiotic amoxicillin was used as a positive control with a final concentration of $1.0 \mathrm{mg} / \mathrm{ml}$.

\subsubsection{Minimum inhibitory concentration (MIC) and Minimum bactericidal concentration (MBC)}

The MIC determination was performed on the microorganisms that were sensitive to the fractions using the broth dilution method [51]. Different concentrations of the extracts were prepared and added to test tubes containing MHB. Bacterial isolates were inoculated into each tube and incubated at $37^{\circ} \mathrm{C}$ for $24 \mathrm{~h}$. The minimum concentrations of the extract which showed no turbidity in the MHB was considered as the MIC.

The MBC was performed to test the viability of microorganisms upon treatment with the compound. The MIC cultures of different concentrations were inoculated on MHA plates and incubated at $37^{\circ} \mathrm{C}$ for $24 \mathrm{~h}$. After incubation the plates were observed for the growth of bacterial colonies and data were recorded. The MBC represents the MHA plate with a minimum concentration of the extract that displays no bacterial growth [52].

\subsection{Molecular docking experiment}

\subsubsection{Ligands and protein preparations}

The 3D structure of acetyleugenol was obtained from the PubChem database (www.pubchem.ncbi.nlm.nih.gov). Other eugenol derivatives-a total of four molecules containing eugenol scaffold-were sketched and converted into 3D structures using ChemDraw (ChemDraw 2017 PerkinElmer Informatics Inc.). All molecules were prepared using the Molecular Operating Environment (MOE) program (www.chemcomp.com, Chemical computing group, Montreal, Canada). Partial charges were added to the compounds using MMFF94 force field before subjecting them to energy minimization to obtain optimized geometries.

For the protein targets preparation, X-ray crystal structures of the M. tuberculosis Serine/Threonine kinases enzymes MtPknE and MtPknB with PDB codes 2H34 and 2FUM, respectively, were retrieved from the protein databank (www.rcsb.org). The structural models were prepared by removing water molecules, adding hydrogen atoms, and applying energy minimization. The binding sites of the MtPknE and MtPknB were predicted using homology modelling. Target protein sequences were submitted to the SWISS-Model server and homolog templates were obtained by BLAST. The template selected for the next experiments was the plant Cipk family of protein kinases (PDB ID: 4CZU). Target proteins and templates were superimposed in the MOE software and the binding sites were searched for using the Site-finder function of the MOE. We purposely selected the binding sites that coincide with the co-crystallized ligand of the template proteins.

\subsubsection{Docking procedure}

The docking experiments were performed using the docking suite of the MOE software. The compound structures were transferred and saved in MOE database. A Gaussian contact surface was drawn around the binding sites of the target protein and the receptor was verified as a receptor and the site as ligand atoms. The Placement method used was Triangle Matcher. The first scoring function was set to London $\mathrm{dG} / \mathrm{MOE}$ and the refinement to force field. The docking process was then started by retaining 100 poses. The final refined poses were ranked by the MM/GBV1 binding free energy estimation. The depiction of results was generated by PyMOL APBS tools and MOE.

\section{Results and Discussion}

\subsection{Isolation and structural elucidation of Eugenol and its antibacterial activity}


A. nilotica (L.) is a medicinal plant that is well-known for its applications in the traditional medicines and has been used extensively to treat various respiratory diseases and other infectious diseases [19-21]. Here, we applied bioactivity guided approach to justify the wide-spread application of A. nilotica (L.) by conducting systematic phytochemical analysis on the leaves and tested its antimicrobial activity against several pathogenic bacteria including E. coli, K. pneumoniae, Proteus sp., P. aeruginosa, S. aureus, S. typhi and S. pneumonia (Figure S1). First, we extracted the chemical compounds from the leaves using methanol and used the methanol extract and marc for further fractionation with $n$-hexane, ethyl acetate and $n$-butanol consecutively (Scheme S1). The ethyl acetate extract was found to be the most effective extract against these bacterial isolates; hence it was selected for further phytochemical analysis in order to isolate the active compound(s). TLC and column chromatography were subsequently applied to fractionate A. nilotica (L.) leaves extracts by using solvent systems containing various ratios of $n$-hexane, methanol and ethyl acetate. Fractions were pooled and categorized based on their similarity in colors and retention factors into 5 sub-fractions named Fractions I-V (Table S1).

Table 1. Antibacterial activity of pure fractions (I-V) from the ethyl acetate extraction of the A. nilotica (L.) leaves.

\begin{tabular}{|c|c|c|c|c|c|c|c|}
\hline \multirow{2}{*}{$\begin{array}{l}\text { Bacterial } \\
\text { isolates }\end{array}$} & \multicolumn{7}{|c|}{ Zone of Inhibition (mm) } \\
\hline & Fraction-I & Fraction-II & Fraction-III & Fraction-IV & Fraction-V & $\begin{array}{c}\text { Methanol } \\
5 \%\end{array}$ & Amox. \\
\hline E. coli & $22.0 \pm 3.00$ & $5.33 \pm 1.52$ & $\overline{\text { n.d }}$ & n.d & $4.33 \pm 2.52$ & n.d & $28.7 \pm 1.15$ \\
\hline К. рпеитопіае & $15.0 \pm 4.58$ & $5.67 \pm 2.31$ & n.d & n.d & $5.33 \pm 1.53$ & n.d & $26.7 \pm 0.58$ \\
\hline Proteus sp. & $29.0 \pm 2.00$ & $4.67 \pm 2.08$ & n.d & n.d & $3.33 \pm 1.53$ & n.d & $30.7 \pm 1.15$ \\
\hline P. aeruginosa & $24.7 \pm 3.51$ & $7.67 \pm 3.06$ & n.d & n.d & $4.33 \pm 1.15$ & n.d & $25.4 \pm 1.53$ \\
\hline S. aureus & $24.3 \pm 4.16$ & $6.00 \pm 1.73$ & n.d & n.d & $3.61 \pm 2.08$ & n.d & $23.0 \pm 1.00$ \\
\hline S. typhi & $28.0 \pm 5.57$ & $2.00 \pm 1.00$ & n.d & n.d & $3.00 \pm 2.00$ & n.d & $28.0 \pm 2.00$ \\
\hline S. pneuтопіа & $26.7 \pm 1.53$ & $5.33 \pm 1.53$ & n.d & n.d & $3.33 \pm 1.52$ & n.d & $21.7 \pm 1.53$ \\
\hline
\end{tabular}

Amox. = Amoxicillin antibiotic (positive control). $5 \%$ Methanol $=$ negative control. $n . d=$ no detected activity. Data presented as mean $\pm \operatorname{std}(n=3)$.

Table 1 shows the antibacterial activity of ethyl acetate fractions that were prepared from the ethyl acetate extract. The most prominent fraction with strong antibacterial activity was fraction I, which exhibits potential activity against all bacterial isolates with noticeable effects against Proteus sp., S. typhi, P. aeruginosa and S. pneumonia. Low activities were observed on fractions II and V and no activities were observed for fractions III and IV. The antibacterial activity for fraction I was similar or even higher in some cases from that of the positive control amoxicillin. Therefore, fraction I was selected for the determination of the MIC and MBC, which further confirmed the antibacterial activity of the fraction (Table 2). These strong antibacterial activities agree well with previous reports on A. nilotica (L.) which can be attributed to the presence of several active phytochemicals in the different parts of $A$. nilotica (L.) such as flavonoids, alkaloids, terpenes and others active ingredients $[19,22,23]$. To elucidate the chemical structure and properties of the active ingredient in fraction I; GC-MS, NMR spectroscopy, FTIR and UV-visible spectroscopy were applied. The GC-MS spectrum indicated that the most prominent compound in fraction I is acetyleugenol with the formula $\mathrm{C}_{12} \mathrm{H}_{14} \mathrm{O}_{3}$-an eugenol derivative (Figure S2). This the first report in the purification of acetyleugenol, to our knowledge, from the leaves of A. nilotica (L.). ${ }^{1} \mathrm{H}$ NMR, ${ }^{13} \mathrm{C}$ NMR and $2 \mathrm{D}$ NMR analyses confirmed the structure of fraction I-acetyleugenol (Figure S3, S4 and S5). The ${ }^{13} \mathrm{C}$ NMR spectral data of fraction I was compared with a reference ${ }^{13} \mathrm{C}$ NMR spectrum of pure acetyleugenol compound [53], as well as theoretically predicted ${ }^{13} \mathrm{C}$ NMR chemical shifts for the acetyleugenol ( $\delta$-ppm) [54]. These comparisons revealed similar chemical shifts for the 12 carbon positions in the backbone of acetyleugenol structure (Table 3) [53,54]. In light of these results along with the GC-MS, we concluded that the major compound of fraction I is an acetyleugenol. Fraction I was homogenous and showed maximum absorption peak $\left(\lambda_{\max }\right)$ at $365 \mathrm{~nm}$ (Figure S6) and its FTIR spectrum indicated the presence of characteristic peaks of well documented eugenol compounds (Figure S7) [55]. Previous results on 
eugenol isolated from other plants indicated significant molecular interactions with Extended Spectrum BetaLactamase enzymes (ESBL) and showed significant antibacterial activity against E. coli and K. pneumoniae [56]. This might explain the strong susceptibility of these bacterial isolates to the acetyeugenol (Table 1,2). Eugenol oil, which contains about $10 \%$ acetyleugenol, has also been shown to exhibit a strong antimicrobial activity against E. coli, S. aureus, and Candida albicans and less activity against Enterococcus fecalis [57]. These studies clearly indicate that acetyleugenol may have broad spectrum antimicrobial activity. Thus, it is important to explore its potential with different drug targets to understand its mechanism of actions. Such knowledge can help to develop novel compounds based on the acetyleugenol or eugenol scaffolds with stronger antimicrobial potential.

Table 2. The Minimum inhibitory concentration (MIC) and the minimal bactericidal concentration (MBC) of fraction I acetyleugenol.

\begin{tabular}{ccl}
\hline Bacterial isolates & $\mathrm{MIC}(\mathrm{mg} / \mathrm{ml})$ & $\mathrm{MBC}(\mathrm{mg} / \mathrm{ml})$ \\
\hline E. coli & 0.31 & 5.00 \\
K. pneumoniae & 1.25 & 10.0 \\
Proteus sp. & 0.63 & 5.00 \\
P. aeruginosa & 1.25 & 5.00 \\
S. aureus & 0.63 & 10.0 \\
S. typhi & 1.25 & n.d \\
S. pneumonia & 2.50 & n.d \\
\hline
\end{tabular}

\subsection{Molecular Docking of Acetyleugenol and its analogues against MtPknB and MtPknE}

To study the effect of acetyleugenol at a molecular level and explore its antimicrobial potential, two important $M$. tuberculosis drug targets were chosen; i) $M t P k n B$ and ii) $M t P k n E ~ S / T$ protein kinases, which play pivotal roles in $M$. tuberculosis development and pathogenesis [34,35]. First, we used the acetyleugenol and conducted molecular docking experiments against the MtPknE protein kinase (PDB ID: 2H34). The results indicated relatively moderate docking score with a binding energy at $-8.53 \mathrm{kcal} / \mathrm{mol}$ (Figure 1). The docking revealed that the acetyleugenol interacts with Ile96 residue of $M t P k n E$ via single hydrogen bond with $2.8 \AA$ which is formed between the carbonyl oxygen of the ester group of acetyleugenol and the hydrogen of the backbone NH group of Ile96 residue (Figure 1a). The two alkyl groups of the acetyleugenol were found to be embedded in a hydrophobic pocket that is formed by several hydrophobic residues including Leu95, Ile96, Ala43, Val22, Val30, Val77, Val156, Phe158, Leu146, and Ile160 (Figure 1b). The vinyl group ( $-\mathrm{CH}=\mathrm{CH} 2)$ of the acetyleugenol is located in a solvent accessible region of the binding cavity and show no interaction with any of the residues in this cavity. This result indicates that the active functional group of the acetyleugenol in the interaction with $M t P k n E$ is the ester carbonyl group. A previous docking study on eugenol showed that both phenolic hydroxyl and methoxy oxygen of eugenol can interact with residues on the active site as demonstrated in the case of $C$. albicans squalene epoxidase [58]. Thus, the acetyleugenol has smaller size relative to the binding cavity of the $M t P k n E$ kinases, which might potentially affect its orientation and binding. This might explains the relatively low binding energy and docking score.

Table 3. Comparison of the ${ }^{13} \mathrm{C}$ NMR spectral data of fraction 1 (acetyleugenol) obtained from the ethyl acetate extract fractionation of $A$. nilotica (L.) with literature and theory.

\begin{tabular}{cccc}
\hline \multirow{2}{*}{ Carbon position } & \multicolumn{3}{c}{${ }^{13}$ C-NMR $(\delta-p p m)$} \\
\cline { 2 - 4 } & Experimental & Literature $^{1}$ & Theoretical $^{2}$ \\
\hline C1 & 138.83 & 139.05 & 138.2 \\
C2 & 166.78 & 150.88 & 151.0 \\
C3 & 52.06 & 112.73 & 112.8 \\
C4 & 146.00 & 138.00 & 137.5 \\
C5 & 108.83 & 120.69 & 120.5
\end{tabular}




\begin{tabular}{cccc} 
C6 & 119.67 & 122.53 & 122.6 \\
C7 & 40.29 & 55.84 & 39.9 \\
C8 & 130 & 40.12 & 137.6 \\
C9 & 100 & 137.06 & 115.6 \\
C10 & 116.19 & 56.2 \\
C11 & 40.53 & 169.28 & 168.6 \\
C12 & 171.83 & 20.71 & 20.8 \\
\hline
\end{tabular}

References: ${ }^{1}$ Lazarevic et al., 2018 [53]; ${ }^{2}$ Banfi and Patinyl, 2008 [54].
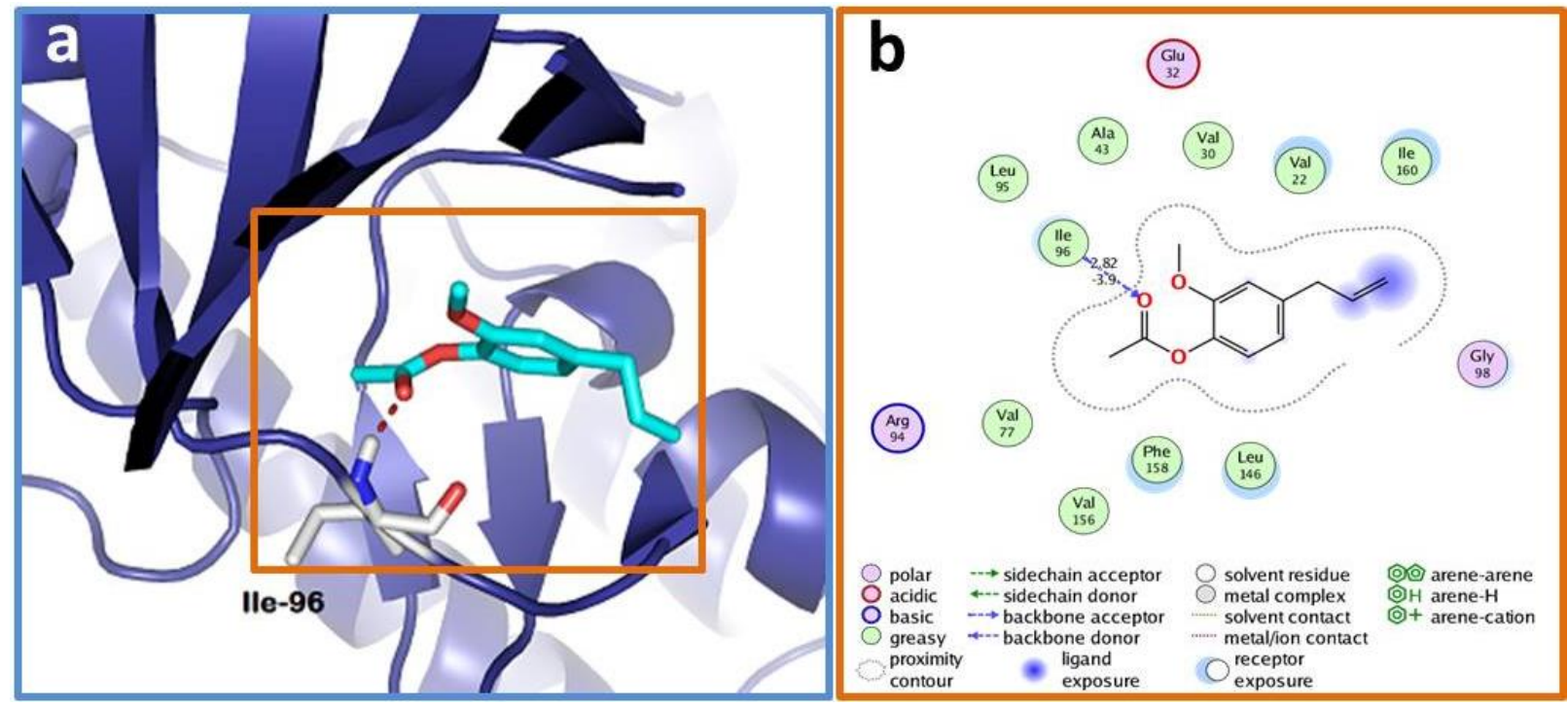

Figure 1. Molecular docking of the eugenol acetate showing (a) the binding interactions in the binding site of $M t P k n E$ kinase. The eugenol acetate shown as stick (cyan), and (b) the 2D representation of the docking generated by MOE software; the amino acid residues are colored according to their chemical nature shown in the key. The 2D views shows the hydrogen bonding network and the Van der Waal contacts with the hydrophobic residues (green).

Table 4. Molecular docking binding free energy of the acetyleugenol compound and its phenyl and indole analogues against $M t P k n B$ and $M t P k n E$ molecular targets.

\begin{tabular}{cccc}
\hline \multirow{2}{*}{ Compound } & \multicolumn{2}{c}{ Binding Energy $(\mathrm{kcal} / \mathrm{mol})$} & \multirow{2}{*}{ Reference } \\
\cline { 2 - 3 } & MtPknE & MtPknB & \\
\hline EUG-1 & -11.08 & -10.46 & This study \\
EUG-2 & -10.29 & -10.19 & This study \\
EUG-3 & -10.05 & -9.64 & This study \\
EUG-4 & -9.25 & -9.35 & This study \\
Acetyleugenol & -8.53 & -7.60 & This study \\
Mitoxantrone & n.a & -10.8 & Ali et al. 2018 [42] \\
\hline
\end{tabular}

In order to optimize the molecular interactions of the acetyleugenol for best orientation and binding in the hydrophobic binding pocket of $M t P k n E$, we performed in silico synthesis for new compounds based on acetyleugenol skeleton modifications by adding either phenyl ring or indole to the ester moiety or the vinyl sites. This gave rise to five acetyleugenol analogs including the acetyleugenol and named compounds 1-5 as depicted in Figure 2. The compounds $\mathbf{1}$ and 3, which were named EUG-1 and EUG-3, respectively, are indole 
analogs with indole group at either the vinyl double bond site (EUG-1) or at the ester site (EUG-3) and similarly the phenyl group was added to make compound 2 (EUG-2) and compound 4 (EUG-4). This is the first report, to our knowledge, to design and study the docking profile of these newly proposed acetyleugenol analogs.

To get insights into the mechanisms of interactions and docking based structure activity relationships of these newly designed compounds M. tuberculosis protein Ser/Thr kinases MtPknB and MtPknE were used as molecular targets [34]. The binding energy values of the docking experiments are listed in Table 4 . The docking results of these compounds appear to be similar between $M t P k n B$ and MtPknE with slightly higher selectivity to $M t P k n E$ than $M t P k n B$. The most selective ligand, ligand-protein affinity, is the EUG-1 which has the lowest binding energy - the best interaction and docking score. The results indicate that the addition of an indole group to the double bond of the vinyl site has significantly improved the docking score in comparison with the acetyleugenol (Table 4). This introduction of an indole ring to the acetyleugenol skeleton in the case of EUG-1 provides an additional hydrogen bond donor via the indole amino group to an existing hydrogen bond acceptor of the ester carbonyl group. The EUG-1 also provides a molecular bulk necessary for Van der Waals and hydrophobic interactions, which further favored the EUG-1 binding energetics and improved the docking scores with both MtPknE and MtPknB (Figure 3). The EUG-1 also reveals similar binding energies to $M t P k n E$ and $M t P k n B$ with $-11.08 \mathrm{kcal} / \mathrm{mol}$ and $-10.46 \mathrm{kcal} / \mathrm{mol}$, respectively (Table 4$)$. A recent study by Ali et al., (2018) has examined several propolis compounds as potential anti-TB drugs including flavonoids pachypodol and pinobanksin-3-(E)-caffeate, which showed lower affinity with docking scores at $-9.1 \mathrm{kcal} / \mathrm{mol}$ binding energy against the $M t P k n B$ comparison to the control inhibitor mitoxantrone, which exhibited a better docking score with $-10.8 \mathrm{kcal} / \mathrm{mol}$ binding energy [42]. When the same propolis were examined against other $M$. tuberculosis enzymes involving MtPank, MtDprE1 and MtKasA, the results indicate similar or better docking scores than that of mitoxantrone [42]. This is a clear indication that the indole analogue EUG-1 of the acetyleugenol is a potential anti-TB that can act against both $M t P k n E$ and $M t P k n B$ kinases. Another compound named IMB-YH-8 has also provided promising clinical trials and was shown to act specifically against the MtPkn enzymes with the most effect against the MtPknB [59].<smiles>COc1cc(CCc2ccc3[nH]ccc3c2)ccc1OC(C)=O</smiles>

(1)<smiles>C=CCc1ccc(OC(=O)c2ccccc2)c(OC)c1</smiles><smiles>COc1cc(CCc2ccccc2)ccc1OC(C)=O</smiles>

(2)<smiles>C=CCc1ccc(OC(C)=O)c(OC)c1</smiles><smiles>C=CCc1ccc(OC(=O)c2ccc3cc[nH]c3c2)c(OC)c1</smiles>

(3)

\begin{tabular}{ll}
\hline$(1)=$ & EUG-1 \\
\hline$(2)=$ & EUG-2 \\
\hline$(3)=$ & EUG-3 \\
\hline$(4)=$ & EUG-4 \\
\hline$(5)=$ & Acetyleugenol \\
\hline
\end{tabular}

Figure 2. The acetyleugenol (5) from A. nilotica (L.) and the in silico synthesized phenyl and indole analogs (this study). 
The docking results indicate that the EUG-1 interacts via its indole amino group and the carbonyl oxygen to form energetically favorable binding affinity with the drug target. It forms three hydrogen bonds with

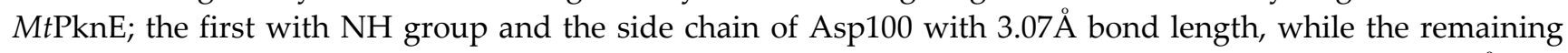
hydrogen bonds are formed between the carbonyl oxygen and the backbone of Leu95 and Ile96 with $3.31 \AA$ and $2.87 \AA$ bond length, respectively (Figure 3a). The EUG-1 interacts with MtPknB via a hydrogen bond that is formed between the indole ring of EUG- 1 and Val95 with $2.67 \AA$ bond length and another hydrogen bond between the EUG-1 carbonyl group with Phe19 with $3.41 \AA$ bond length. Furthermore, the EUG-1 also interacts with $M t P k n B$ via a relatively weak $\mathrm{H}-\pi$ interaction between its indole pyrrole moiety and a hydrogen of Leu17 (Figure $3 b$ ).
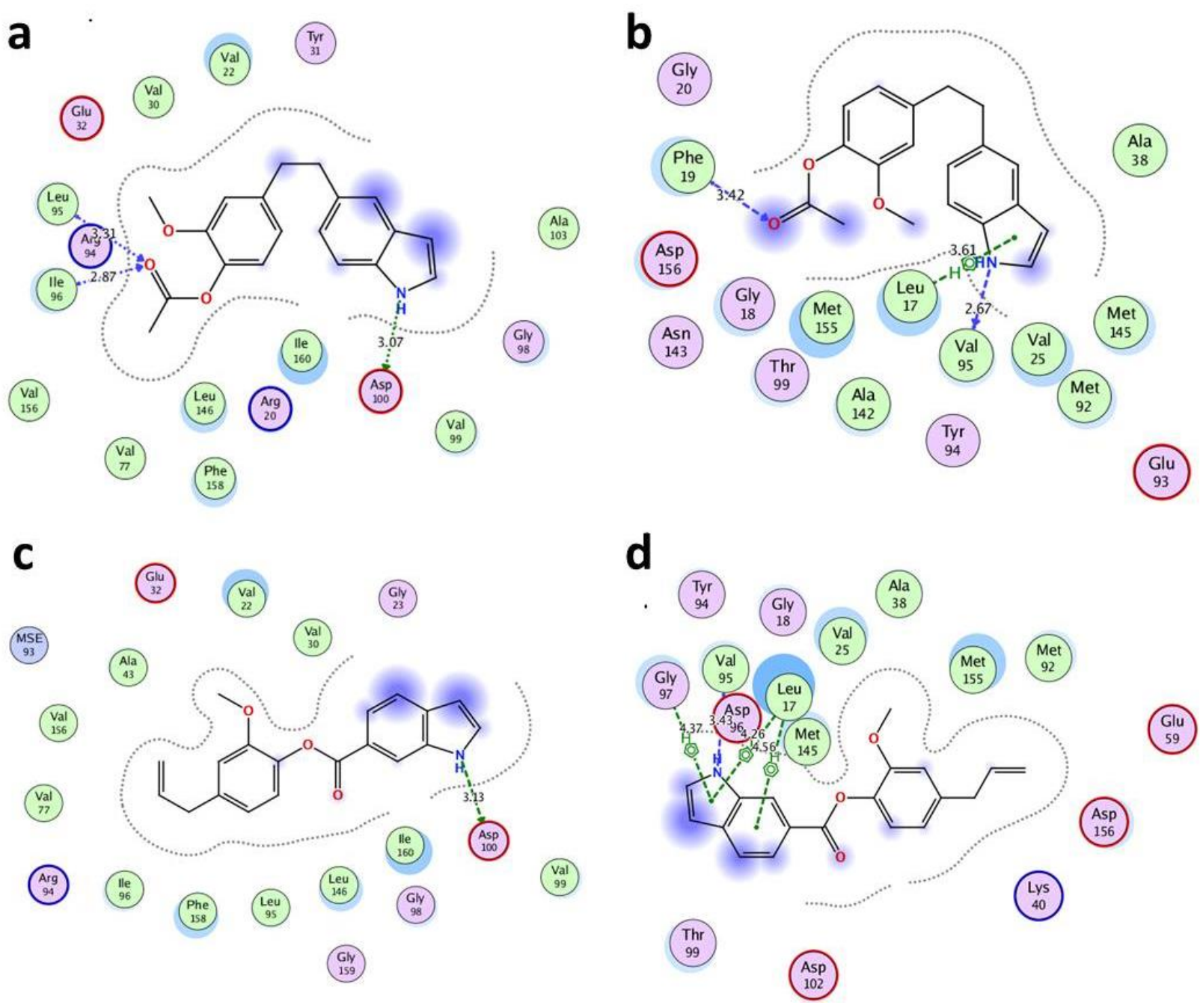

Figure 3. Binding interactions of eugenol-indole analogs with MtPknE and MtPknB S/T kinases; a) EUG-1 with $M t P k n E$, b) EUG-1 with $M t P k n B$, c) EUG-3 with $M t P k n E$ and d) EUG-3 with $M t P k n B$.

EUG-3, which has an indole group attached to the ester end of acetyleugenol, reveals improved docking scores relative to the acetyleugenol compound for both enzymes $M t P k n E$ and $M t P k n B$ with $-10.29 \mathrm{kcal} / \mathrm{mol}$ and $-10.19 \mathrm{kcal} / \mathrm{mol}$ binding energy, respectively (Table 1). These values are only slightly higher than that of mitoxantrone standard inhibitor for $M t P k n B$ that scores $-10.8 \mathrm{kcal} / \mathrm{mol}$ with $0.8 \mu \mathrm{M}$ IC50 [42]. Unlike EUG-1, the carbonyl group of EUG-3 doesn't interact with any of the amino acid residues in the binding pocket owing most likely to the shielding provided by the bulky indole moiety that prevents the carbonyl from approaching any nearby residues as illustrated in the case of MtPknE (Figure 5c). EUG-3 interacts with MtPknB via one hydrogen bond that is formed between the amino group of the indole pyrrole and Val95. Additionally, we observed three weak $\mathrm{H}-\pi$ interactions among the EUG-3 aromatic benzyl and pyrrole rings and the enzyme 
Gly97, Leu17 and Met145 (Figure 5d). In contrast, the phenyl analogs EUG-2 and EUG-4, which have a phenyl ring attached either to the ester group site or to the double bond of the vinyl group of the acetyleugenol skeleton, show weaker binding affinities for both enzymes comparing with that of the EUG-1 and EUG-3 (Table 1). EUG-2 has slightly better binding affinity comparing to EUG-4 with $-10.05 \mathrm{kcal} / \mathrm{mol}$ binding energy for $M t P k n E$ and $-9.64 \mathrm{kcal} / \mathrm{mol}$ for $M t P k n B$. Altogether, all of the modifications applied in the present study have improved the binding affinity and docking score when compared with our starting compound acetyleugenol. Generally, replacing the methyl group of the ester moiety by indole rather than phenyl group has robustly influenced the binding energetics and interactions with the hydrophobic pocket of the enzymes. On the other hand, the choice of the site of substitution has also played a paramount role in the improvement of the binding affinity, hence the docking scores especially with the MtPknE target. Therefore, these compounds are more likely to be good leads for the discovery of potential anti-tuberculosis drugs.

\section{Conclusions}

Here, we isolated and elucidated the molecular structure of acetyleugenol from the leaves of A. nilotica (L.) and confirmed its antibacterial potential against seven clinical isolates. We further demonstrated for the first time the potential anti-TB of the acetyleugenol analogs which were designed, in silico synthesized and used for molecular docking experiments against $M$. tuberculosis Ser/Thr protein kinases-the MtPknE and MtPknB. Our docking results indicate that the indole analogs EUG-1 and EUG-3 are potential inhibitors targeting these kinases with promising outcome. The effective interactions of EUG-1 and EUG-3 with both targets suggest a dual mechanism of actions for these compounds and proposing a possible interference with the pathogenesis and cell division of $M$. tuberculosis at different stages based on the functions of PknE and PknB in M. tuberculosis development. This urgently require chemical synthesis and more comprehensive in vivo and in vitro experiments to understand their mechanism of actions against $M$. tuberculosis or other mycobacterial models such as M. smegmatis to enable designing an effective anti-TB drug. The present study also encourages for further investigation of other acetyleugenol derivatives against these kinases or other potential anti-TB targets.

Supplementary Materials: The following are available online at www.mdpi.com/xxx/s1, Scheme 1: extraction and fractionation of the methanol crude extract of the A. nilotica leaves, Figure S1: phytochemical and antibacterial screening, Figure S2: GC-MS analysis of acetyleugenol, Figure S3: 13C NMR spectrum of acetyleugenol, Figure S4: ${ }^{1} \mathrm{H}$ NMR and the DEPT experiments of acetyleugenol, Figure S5: 2D COSY correlation of acetyleugenol, Figure S6: UV-visible spectrum of acetyleugenol, Figure S7: FT-IR analysis of the acetyleugenol, Table S1: thin layer chromatography profile.

Author Contributions: Conceptualization, F.H.M.K. and A.M.I; proposed and designed compounds, F.H.M.K.; conducting experiments, A.A., T.A.A., and A.E.A.; writing-original draft preparation, F.H.M.K.; writing-review and editing, A.A., T.A.A., A.M.I., and F.H.M.K. All authors have read and agreed to the published version of the manuscript.

Funding: This research received no external funding.

Acknowledgments: The authors thank the staff of the Department of Biological Sciences, Usmanu Danfodiyo University for technical support.

Conflicts of Interest: The authors declare no conflict of interest.

\section{References}

1. MacNeil, A.; Glaziou, P.; Sismanidis, C.; Maloney, S.; Floyd, K. Global Epidemiology of Tuberculosis and Progress Toward Achieving Global Targets- 2017. MMWR Morb. Mortal. Wkly Rep. 2019, 68, 263-266. doi:10.15585/mmwr.mm6811a3.

2. Seung KJ.; Keshavjee S.; Rich ML. Multidrug-resistant tuberculosis and extensively drug-resistant tuberculosis. Cold Spring Harb. Perspect. Med. 2015, 5:a017863. doi:10.1101/cshperspect.a017863.

3. Sakamoto, H.; Lee, S.; Ishizuka, A.; Hinoshita, E.; Hori, H.; Ishibashi, N.; Komada, K.; Norizuki, M.; Katsuma, Y.; Akashi, H.; Shibuya, K. Challenges and opportunities for eliminating tuberculosis - leveraging political momentum of the UN high-level meeting on tuberculosis. BMC Public Health 2019, 19, 76. doi:10.1186/s12889-019-6399-8.

4. World Health Organization (WHO). Available online: https://www.who.int/news-room/fact-sheets/detail/tuberculosis (accessed on 24 March 2020). 
5. $\quad$ Liang, L.; Wu, Q.; Gao, L.; Hao, Y.; Liu, C.; Xie, Y.; Sun, H.; Yan, X.; Li, F.; Li, H.; Fang, H.; Ning, N.; Han, L. Factors contributing to the high prevalence of multidrug-resistant tuberculosis: a study from China. Thorax 2012, 67, 632-638. doi:10.1136/thoraxjnl-2011-200018.

6. Gandhi, N.R.; Moll, A.; Sturm, A.W.; Pawinski, R.; Govender, T.; Lalloo, U.; Zeller, K.; Andrews, J.; Friedland, G. Extensively drug-resistant tuberculosis as a cause of death in patients co-infected with tuberculosis and HIV in a rural area of South Africa. Lancet 2006, 368, 1575-1580. doi:10.1016/S0140-6736(06)69573-1.

7. Glaziou, P.; Sismanidis, C.; Floyd, K.; Raviglione, M. Global epidemiology of tuberculosis. Cold Spring Harb. Perspect. Med. 2015, 5, a017798. doi:10.1101/cshperspect.a017798

8. Zhang, Y.; Heym, B.; Allen, B.; Young, D.; Cole, S. The catalase-peroxidase gene and isoniazid resistance of Mycobacterium tuberculosis. Nature 1992, 358:591-593. doi:10.1038/358591a0.

9. Rawat, R.; Whitty, A.; Tonge, P.J. The isoniazid-NAD adduct is a slow, tight-binding inhibitor of InhA, the Mycobacterium tuberculosis enoyl reductase: Adduct affinity and drug resistance. Proc. Natl. Acad. Sci. USA 2003, 100, 13881-13886. doi:10.1073/pnas.2235848100.

10. Palomino, JC.; Martin, A. Drug resistance mechanism in Mycobacterium tuberculosis. Antibiotics 2014, 3, 317-340. doi:10.3390/antibiotics3030317.

11. Maruri, F.; Sterling, T.R.; Kaiga, A.W.; Blackman, A.; van der Heijden, Y.F.; Mayer, C.; Cambau, E.; Aubry, A. A systematic review of gyrase mutations associated with fluoroquinolone-resistant Mycobacterium tuberculosis and a proposed gyrase numbering system. J. Antimicrob. Chemother. 2012, 67, 819-831. doi:10.1093/jac/dkr566.

12. Reeves, A.Z.; Campbell, P.J.; Sultana, R.; Malik, S.; Murray, M.; Plikaytis, B.B.; Shinnick, T.M.; Posey, J.E. Aminoglycoside Cross-Resistance in Mycobacterium tuberculosis due to Mutations in the $5^{\prime}$ untranslated region of whiB7. Antimicrob. Agents Chemother. 2013, 57, 1857-1865. doi:10.1128/AAC.02191-12.

13. Asadbeigi, M.; Mohammedi, T.; Rafieian-Kopaei, M.; Saki, K.; Bahmani, M.; Delfan, M. Traditional effects of medicinal plants in the treatment of respiratory diseases and disorders: an ethnobotanical study in the Urmia. Asian Pas. J. Trop. Med. 2014, 7, S364-S368. doi:10.1016/S1995-7645(14)60259-5.

14. Cragg, G.M.; Newman, D.J. Natural products: a continuing source of novel drug leads. Biochim Biophys. Acta 2013, 1830, 3670-3695. doi:10.1016/j.bbagen.2013.02.008.

15. Atanasov, A.G.; Waltenberger, B.; Pferschy-Wenzig, E.-M.; Linder, T.; Wawrosch, C.; Uhrin, P.; Temml, V.; Wang, L.; Schwaiger, S.; Heiss, E.H.; Rollinger, J.M.; Schuster, D.; Breuss, J.M.; Bochkov, V.; Mihovilovic, M.D.; Kopp, B.; Bauer, R.; Dirsch, V.M.; Stuppner, H. Discovery and resupply of pharmacologically active plant-derived natural products: a review. Biotechnol. Adv. 2015, 33, 1582-1614. doi:10.1016/j.biotechadv.2015.08.001.

16. Ekor, M. The growing use of herbal medicines: issues relating to adverse reactions and challenges in monitoring safety. Front. Pharmacol. 2014, 4, 177. doi:10.3389/fphar.2013.00177.

17. Van Wyk, B.-E. The potential of South African plants in the development of new medicinal products. South Afr. J. Bot. 2011, 77, 812-829. doi:10.1016/j.sajb.2011.08.011.

18. Semenya, S.S.; Maroyi, A. Data on medicinal plants used to treat respiratory infections and related symptoms in South Africa. Data Brief 2018, 21, 419-423. doi:10.1016/j.dib.2018.10.012.

19. Karar, M.G.E.; Kuhnert, N. Herbal drugs from Sudan: traditional uses and phytoconstituents. Pharmacogn. Rev. 2017, 11,83-103. doi:10.4103/phrev.phrev_15_15.

20. Hussein, G.; Miyashiro, H.; Nakamura, N.; Hattori, M.; Kakiuchi, N.; Shimotohno, K. Inhibitory effects of Sudanese medicinal plant extracts on hepatitis C virus (HCV) protease. Phytother. Res. 2000, 14, 510-6. doi:10.1002/10991573(200011)14:7<510::AID-PTR646>3.0.CO;2-B.

21. Alli, L.A.; Adesokan, A.A.; Salawu, A.O. Antimalarial activity of fractions of aqueous extract of Acacia nilotica root. J. Intercult. Ethnipharmacol. 2018, 5, 180-185. doi:10.5455/jice.20160331064817.

22. Singh, R.; Singh, B.; Singh, S.; Kumar, N.; Kumar, S.; Arora, S. Anti-free radical activities of kaempferol isolated from Acacia nilotica (L.) Willd. Ex. Del. Toxicol. In Vitro. 2008, 22:1965-70. doi:10.1016/j.tiv.2008.08.007.

23. Kaur, K.; Michael, H.; Arora, S.; Härkönen, P.; Kumar, S. In vitro bioactivity-guided fractionation and characterization of polyphenolic inhibitory fractions from Acacia nilotica(L.). Willd. ex Del. J. Ethnopharmacol. 2005, 99, 353-60. doi:10.1016/j.jep.2005.01.040.

24. Saini, M.L.; Saini, R.; Roy, S.; Kumar, A. Comparative pharmacognostical and antimicrobial studies of Acacia species (Mimosaceae). J. Med. Plants Res. 2008, 2, 378-86.

25. Musa, M.S.; Abdelrasool, F.E.; Elsheikh, E.A.; Ahmed, L.A.; Mahmoud, A.L.; Yagi, S.M. Ethnobotanical study of medicinal plants in the Blue Nile State, South-eastern Sudan. J. Med. Plants Res. 2011, 5, 4287-97. 
26. Grush, J.; Noakes, D.L.; Moccia, R.D. The efficacy of clove oil as an anesthetic for the zebrafish, Danio rerio (Hamilton). Zebrafish 2004, 1, 46-53. doi:1089/154585404774101671.

27. Mallavarapu, G.R.; Ramesh, S.; Chandrasekhara, R.S.; Rajeswara Rao, B.R.; Kaul, P.N.; Bhattacharya, A.K. Investigation of the essential oil of cinnamon leaf grown at Bangalore and Hyderabad. Flavour Fragr. J. 1995, 10, 239242. doi:10.1002/ffj.2730100403.

28. Clarke, S. Families of compounds that occur in essential oils. In Essential Chemistry for Aromatheraphy (2nd ed.), Churchill Livingstone, 2008, pages 41-77. doi:10.1016/B978-0-443-10403-9.00003-0.

29. Bai, S.; Seasotiya, L.; Malik, A.; Bharti, P.; Dalal, S. GC-MS analysis of chloroform extract of Acacia nilotica L. leaves. J. Pharmacog. Phytochem. 2014, 2:79-82.

30. Batiha, G.E.S.; Alkazmi, L.M.; Wasef, L.G.; Beshbishy, A.M.; Nadwa, E.H.; Rashwan, E.K. Syzygium aromaticum L. (Myrtaceae): traditional uses, bioactive chemical constituents, pharmacological and toxicological activities. Biomolecules 2020, 10, 202. doi:10.3390/biom100202002.

31. Barboza, J.N.; da Silva Maia Bezerra Filho, C.; Silva, R.O.; Medeiros, J.V.R.; de Sousa, D.P. An Overview on the antiinflammatory potential and antioxidant profile of eugenol. Oxid. Med. Cell. Longev. 2018, 2018:3957262. doi:10.1155/2018/3957262.

32. de Almeida, A.L.; Caleffi-Ferracioli, R.; de L Scodro, R.B.; Baldin, V.P.; Montaholi, D.C.; Spricigo, L.F.; NakamuraVasconcelos, S.S.; Hegeto, L.A.; Sampiron, E.G.; Costacurta, G.F.; dos S Yamazaki, D.A.; de F Gauze, G.; Siqueira, V.L.D.; Cardoso, R.F. Eugenol and derivatives activity against Mycobacterium tuberculosis, nontuberculous mycobacteria and other bacteria. Future Microbiol. 2019, 14, 331-344. doi:10.2217/fmb-2018-0333.

33. Rohane, S.H.; Chauhan, A.J.; Fuloria, N.K.; Fuloria, S. Synthesis and in vitro antimycobacterial potential of novel hydrazones of eugenol. Arab. J. Chem. 2020, 13, 4495-4504. doi:10.1016/j.arabjc.2019.09.004.

34. Khan, M.Z.; Kaur, P.; Nandicoori, V.K. Targeting the messengers: Serine/threonine protein kinases as potential targets for antimycobacterial drug development. IUBMB Life 2018, 70, 889-904. doi:10.1002/iub.1871.

35. Mori, M.; Sammartino, J.C.; Costantino, L.; Gelain, A.; Meneghetti, F.; Villa, S.; Chiarelli, L.R. An overview on the potential antimycobacterial agents targeting serine/threonine protein kinases from Mycobacterium tuberculosis. Curr. Top. Med. Chem. 2019, 19, 646-661. doi:10.2174/1568026619666190227182701.

36. Wehenkel, A.; Bellinzoni, M.; Grana, M.; Duran, R.; Villarino, A.; Fernandez, P.; Andre-Leroux, G.; England, P.; Takiff, H.; Cervenansky, C.; Cole, S.T.; Alzari, P.M. Mycobacterial Ser/Thr protein kinases and phosphatases: physiological roles and therapeutic potential. Biochim. Biophys. Acta, 2008, 1784, 193-202. doi:10.1016/j.bbapap.2007.08.006.

37. Kang, C.M.; Abbott, D.W.; Park, S.T.; Dascher, C.C.; Cantley, L.C.; Husson, R.N. The Mycobacterium tuberculosis serine/threonine kinases PknA and PknB: substrate identification and regulation of cell shape. Genes Dev. 2005, 19, 1692-1704. doi:10.1101/gad.1311105.

38. Hatzios, S.K.; Baer, C.E.; Rustad, T.R.; Siegrist, M.S.; Pang, J.M.; Ortega, C.; Alber, T.; Grundner, C.; Sherman, D.R.; Bertozzi, C.R. Osmosensory signaling in Mycobacterium tuberculosis mediated by a eukaryotic-like Ser/Thr protein kinase. Proc. Natl. Acad. Sci. USA 2013, 110, E5069-E5077. doi:10.1073/pnas.1321205110.

39. Jayakumar, D.; Jr Jacobs, W.R.; Narayanan, S. Protein kinase E of Mycobacterium tuberculosis has a role in the nitric oxide stress response and apoptosis in a human macrophage model of infection. Cell. Microbiol. 2008, 10,365-374. doi:10.1111/j.1462-5822.2007.01049.x.

40. Kumar, D.; Narayanan, S. PknE, a serine/threonine kinase of Mycobacterium tuberculosis modulates multiple apoptotic paradigms. Infect. Genet. Evol. 2012, 12, 737-747. doi:10.1016/j.meegid.2011.09.008.

41. Gupta, V.K.; Kumar, M.M.; Bisht, D.; Kaushik, A. Plants in our combating strategies against Mycobacterium tuberculosis: progress made and obstacles met. Pharm Biol. 2017, 55, 1536-1544. doi:10.1080/13880209.2017.1309440.

42. Ali, M.T.; Blicharska, N.; Shilpi, J.A.; Seidel, V. Investigation of the anti-TB potential of selected propolis constituents using a molecular docking approach. Sci. Rep. 2018, 8, 12238. Doi: 10.1038/s41598-018-30209-y.

43. Young, T.A.; Delagoutte, B.; Endrizzi, J.A.; Falick, A.M.; Alber, T. Structure of Mycobacterium tuberculosis PknB supports a universal activation mechanism for Ser/Thr protein kinases. Nat. Struct. Biol. 2003, 10, $168-174$. doi:10.1038/nsb897.

44. Gay, L.M.; Ng, H.L.; Alber, T. A conserved dimer and global conformational changes in the structure of apo-PknE Ser/Thr protein kinase from Mycobacterium tuberculosis. J. Mol. Biol. 2006, 360, 409-420. doi:10.1016/j.jmb.2006.05.015.

45. Greenstein, A.E.; Echols, N.; Lombana, T.N.; King, D.S.; Alber, T. Allosteric activation by dimerization of the PknD receptor Ser/Thr protein kinase from Mycobacterium tuberculosis. J. Biol. Chem. 2007, 282, 11427-11435. doi:10.1074/jbc.m610193200.

46. Cannell, R.J.P. (Ed.). Natural Products Isolation, Methods in Biotechnology 4, Humana Press, Totowa, NJ, 1998. 
47. Patil, P.; Shettigar, R. An advancement of analytical techniques in herbal research. J. Adv. Sci. Res. 2010, 1, 8-14.

48. Baker, P.J.; Slots, J.; Genco, R.J.; Evans, R.T. Minimal inhibitory concentrations of various antimicrobial agents for human oral anaerobic bacteria. Antimicrob. Agents Chemother. 1983, 24, 420-424. doi:10.1128/aac.24.3.420.

49. Dahiya, P.; Purkayastha, S. Phytochemical screening and antimicrobial activity of some medicinal plants against multi-drug resistant bacteria from clinical isolates. Indian J. Pharm. Sci. 2012, 74, 443-450. doi:10.4103/0250-474X.108420.

50. Koua, F.H.M.; Babiker, H.A.; Halfawi, A., Ibrahim, R.O.; Abbas, F.M.; Elgaail, E.I.; Khalafallah, M.M. Phytochemical and biolofical study of Striga hermonthica (Del.) Benth callus and intact plant. Res. Parma. Biotechnol. 2011, 3, 85-92.

51. Vollekova, A.D.; Kostalova, R.; Sochorova, R. Isoquinoline alkaloids from Mahonia aquifolium stem bark is active against Malassezia sp. Folia. Microbiol. 2001, 46, 107-111. doi:10.1007/bf02873586.

52. Taylor, P.C.; Schoenknecht, F.D.; Sherris, J.C.; Linner, E.C. Determination of minimum bactericidal concentrations of oxacillin for Staphylococcus aureus: influence and significance of technical factors. Antimicrob. Agents Chemother. 1983, 23, 142-150. doi:10.1128/aac.23.1.142.

53. Lazarevic, J.; Kolarevic, A.; Stojanovic, G.; Smelcerovic, A.; Ciuffred, P.; Santaniello, E. Synthesis, antimicrobial activity and in silico studies on eugenol eters. Acta Chim. Slov. 2018, 65, 801-810. doi:10.17344/acsi.2018.4380.

54. Banfi, D.; Patiny, L. www.nmrdb.org: Resurrecting and processing NMR spectra on-line. Chimia 2008, 62, $280-281$.

55. Carrasco, A.H.; Espinoza, C.L.; Cardile, V.; Gallardo, C.; Cardona, W.; Lombardo, L.; Catalán, M.K.; Cuellar, F.M.; Russo, A. Eugenol and its synthetic analogues inhibit cell growth of human cancer cells (Part I). J. Braz. Chem. Soc. 2008, 19, 543-548. doi:10.1590/S0103-50532008000300024.

56. Dhara, L.; Tripathi, A. Antimicrobial activity of eugenol and cinnamaldehyde against extended spectrum beta lactamase producing enterobacteriaceae by in vitro and molecular docking analysis. Eur. J. Integr. Med. 2013, 5, 527536. doi:10.1016/j.eujim.2013.08.005.

57. Thosar, N.; Basak, S.; Bahadure, R.N.; Rajurkar, M. Antimicrobial efficacy of five essential oils against oral pathogens: an in vitro study. Eur. J. Dent. 2013, 7, S071-S077. doi:10.4103/1305-7456.119078.

58. Hipólito, T.M.M.; Bastos, G.T.L; Barbosa, T.W.L.; de Souza, T.B.; Coelho, L.F.L.; Dias, A.L.T.; Rodriguez, I.C., Dos Santos, M.H.; Dias, D.F.; Franco, L.L.; Carvalho, D.T. Synthesis, activity, and docking studies of eugenol- based glucosides as new agents against Candida sp. Chem. Biol. Drug Des. 2018, 92, 1514-1524. doi:10.1111/cbdd.13318.

59. Xu, J.; Wang, J.; Zhou, J.; Xu, C.-L.; Huang, B.; Xing, Y.; Wang, B.; Luo, R.; Wang, Y.-C.; You, X.-F.; Lu, Y.; Yu, L.-Y. A novel protein kinase inhibitor IMB-YH-8 with anti-tuberculosis activity. Sci. Rep. 2017, 7, 5093. doi:10.1038/s41598-01704108-7. 